\title{
FLUOROPHOTOMETRIC APPARATUS FOR THE OBJECTIVE DETERMINATION OF FLUORESCENCE IN THE ANTERIOR CHAMBER OF THE LIVING EYE*
}

\author{
BY
}

\author{
MAURICE LANGHAM AND KENNETH C. WYBAR
}

From the Institute of Ophthalmology, London

For many years considerable attention has been focused on the problem of the origin and circulation of the aqueous humour under normal and pathological conditions. One of the methods applied to this study is the detection within the eye of dyes which enter the anterior chamber across the bloodaqueous barrier-a method first suggested by Ehrlich (1882) when he observed that fluorescein injected intravenously passes into the aqueous humour. Ehrlich anticipated that the use of this dye would promote a deeper understanding of the problem, and many ways have since been devised of determining the presence of fluorescein in the eye of the living animal. In the present paper an instrument is described which permits an objective determination of the degree of fluorescence under experimental and clinical conditions.

\section{Apparatus}

The main component parts and optical principles of the fluorophotometer are illustrated in Figs $1(a)$ and $1(b)$. Light from the anterior chamber passes through a 1-inch objective lens, and is transmitted partly through the monocular microscope tube and eyepiece to the observer, and partly, after reflection, to the cathode area of a photo-electric multiplier tube (RCA 931A). The reflector lying immediately behind the objective lens comprises a completely silvered mirror mounted on a pivot arm in such a way that the angle between it and the incident light can be adjusted to ensure reflection of the light from the anterior chamber to the centre of the cathode area of the photo-electric multiplier tube; this is determined by replacing the case containing the tube by a holder with a ground glass screen on which the reflected light can be viewed. Once the mirror has been set at the correct angle it may be fixed by means of a small locking screw. The size of the image falling on the photosensitive area of the photo-electric multiplier tube may be limited by the introduction at the focal plane of a diaphragm varying in size and shape with the area of the anterior chamber to be studied. Without the introduction of a diaphragm the effective area of the field of view is $15 \mathrm{~mm}$. in diameter, equivalent to an actual field of view of $4.54 \mathrm{~mm}$., the objective lens giving a linear magnification of 2.75 . In practice, however, a diaphragm 1-2 mm. in diameter is used to limit the field to a narrow area of the anterior chamber, which in the rabbit is approximately $3 \mathrm{~mm}$. in depth.

The photo-electric multiplier tube is a high vacuum photocell with a maximal response at $4000 \AA$. The photo-electric current produced at the cathode is multiplied many times by secondary emission occurring at successive cathodes within the valve. The tube used in the apparatus is supplied by a series of H.T. dry batteries, the voltage of which is equally distributed by means of a group of nine micro-resistances incorporated

*Received for publication July 30, 1953. 
FIG. 1. (a).-Component parts of fluorophotometer.
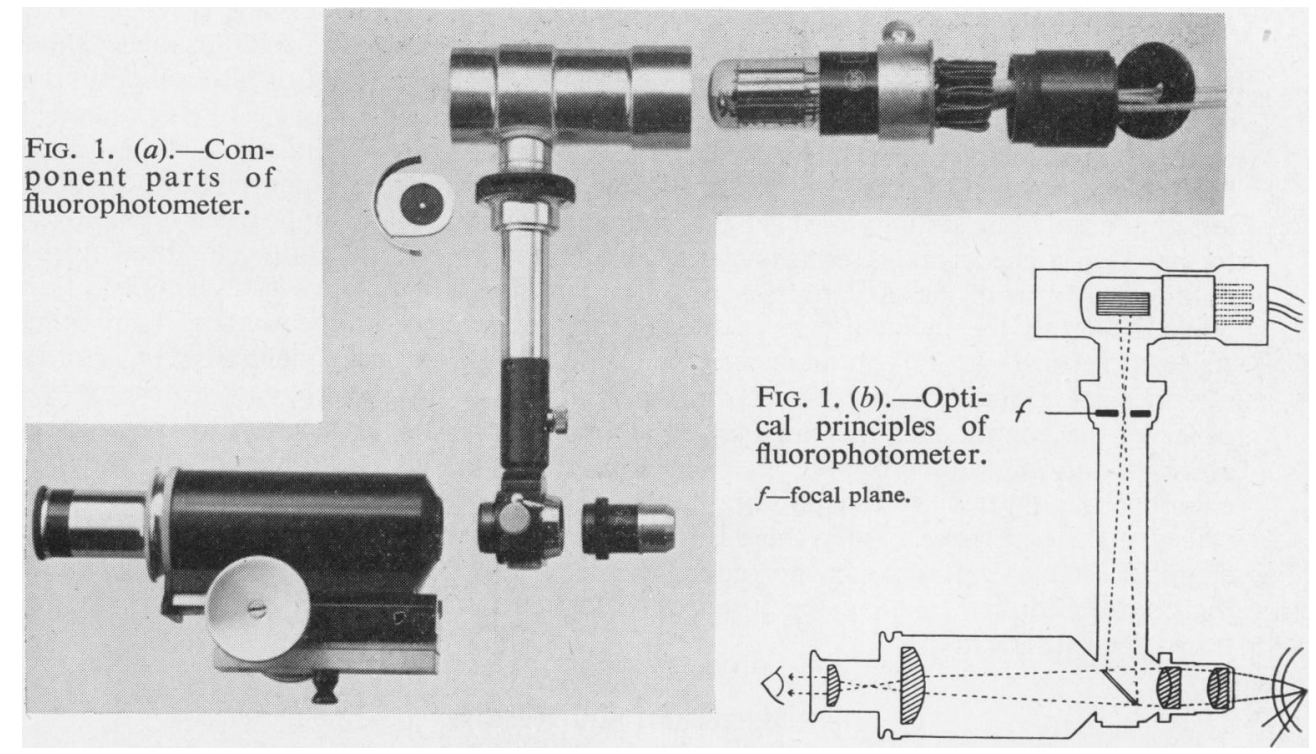

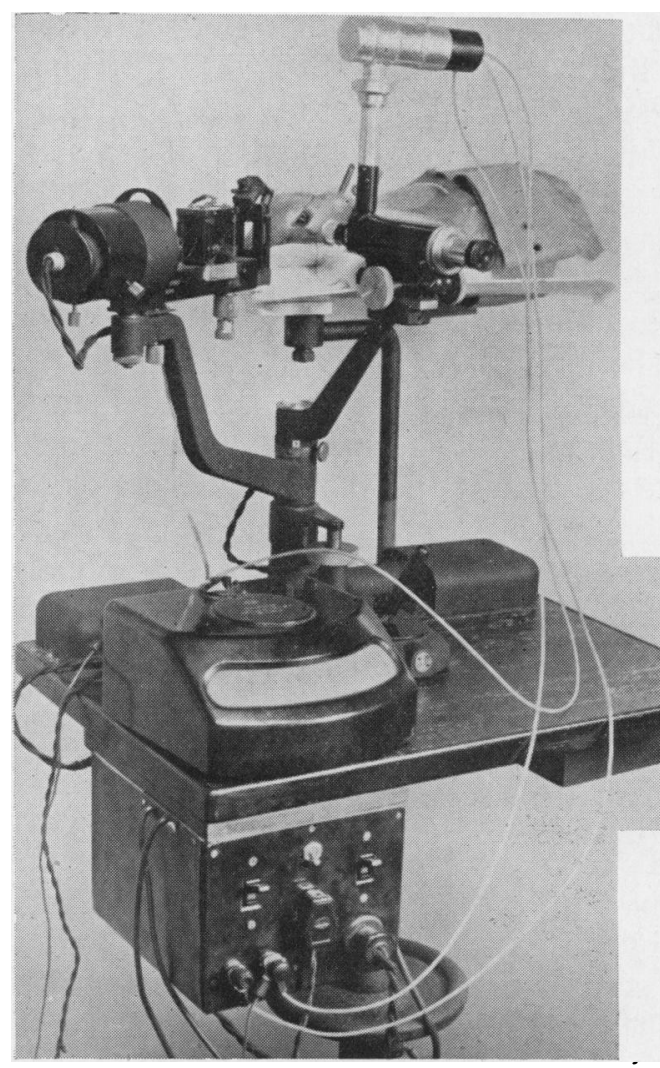

FIG. 2.-Fluorophotometer mounted on Haag-Streit slit lamp. into the base of the photomultiplier case to the nine secondary cathodes. In normal operating conditions the resultant output current from this tube is a linear function of the exciting illumination. The output of the photomultiplier tube is recorded directly on a Cambridge damped galvanometer. The illumination system comprises a 6 v. $3 \cdot 5$ amp. bulb with a vertical filament energized through a constant voltage transformer from the mains supply. Where necessary a second circuit is introduced to provide increased illumination by supplying the bulb with a further 2 volts.

There are two refinements which may be incorporated into the instrument. First, a colour filter (Ilford 605 or 625) may be placed in front of the cathode area to allow preferential transmission of fluorescent light; secondly, crossed polaroids may be fitted to reduce the effect of reflected and scattered light. In animal studies, however, these modifications are not found to be necessary.

The fluorophotometer is mounted on a conventional Haag-Streit slit 
lamp wherein the binocular microscope is replaced by a monocular one (Fig. 2). The observer views the anterior chamber through the microscope, and adjusts the angle between the arm carrying the microscope and that carrying the light to give the optimum view of the anterior chamber. This angle, which is normally about $60^{\circ}$, is maintained throughout the experiment. The focal vertical beam of light is adjusted to the required intensity by varying the width of the slit at the beginning of the experiment, and is then left unaltered. The stability of this light is generally adequate, but a photocell is employed to maintain a check on the constancy of the illumination. The photocell, fitted to the illuminating arm of the slit lamp (see Fig. 2), comprises a plane glass slide inclined at an angle of $45^{\circ}$ to the incident arm of the slit lamp, a fraction of the incident light being reflected vertically to fall on a barrier type of photocell. A target, consisting of a series of concentric rings, is incorporated into the eye-piece, so that before each recording the observer may check that the rings are in a constant position in relation to the corneaaqueous and lens-aqueous barriers. This ensures that the same area of the anterior chamber is analyzed by the photomultiplier tube during each recording. In the later stages of the experiment, when there is a high concentration of fluorescein in the anterior chamber, it may be necessary to reduce the intensity of the fluorescent light reaching the photomultiplier tube by the insertion of a neutral filter of known optical density below the cathode area.j

\section{Discussion and Results}

It is becoming increasingly clear that a satisfactory solution to the problems of intra-ocular dynamics, both in the experimental and clinical fields, will not be achieved until a method is devised for the accurate determination of the concentration of substances in the intact anterior chamber of the living animal. Up to the present time these dynamic studies have followed a similar pattern. The concentration of a substance in the plasma is raised and its time-course compared with the concentration in the aqueous humour removed from each eye at predetermined times. The limitations of this method, only too well recognized by those who have used it, follow from the fact that withdrawal of aqueous humour leads to a marked increase in the permeability of the blood-aqueous barrier with the formation of a plasmoid aqueous, so that only two points on the time-course of the aqueous humour can be obtained for any one experiment. This drawback can be minimized to a certain extent by pooling the results obtained from numerous animals; the work of Palm (1947) on the entry of radioactive phosphate, and of Maurice (1951) on the entry of radioactive sodium are excellent examples of what may be achieved, although the inherent variation between different animals, even between members of the same species, limits the interpretation of such a composite study.

A modification of this experimental method is seen in the work of Bárány and Kinsey (1949), who utilized the phenomenon of the rapid renal excretion of certain compounds to study the rate of their disappearance from the aqueous humour. For example, by raising the concentration of $p$-aminohippuric acid in the plasma for a sufficient length of time, a stage may be reached when the concentration of the tracer substances in the aqueous humour exceeds that in the plasma. The rate of decay of the substance in the aqueous 
humour is then determined by examining the aqueous removed from the other eye after a certain interval. The results obtained by this method are susceptible to mathematical analysis and important conclusions on the rate of flow of aqueous humour have been drawn.

In considering the problem of diffusion in the eye, it is known that a substance may pass into the anterior and posterior chambers from the uveal tract across the blood-aqueous barrier, but it is not justifiable to assume that in the early stages the substance exists in the two chambers in equal concentrations, although, in the presence of the circulation and flow of aqueous humour, the substance may eventually become evenly distributed throughout its volume. The substance will continue to accumulate in the aqueous humour until a state of dynamic equilibrium* is reached between its concentration in the plasma and in the aqueous humour, the so-called steady state, at which point the rate of accumulation is zero. After the steady state has been attained, the rate of replacement of the substance from the blood falls below its rate of disappearance from the aqueous, and its concentration in the aqueous humour is lowered until it is completely eliminated. During this diminishing phase the substance may once more become unevenly distributed throughout the aqueous humour.

Several methods have been described previously for assessing the concentration of fluorescent dyes in the aqueous humour of the living eye after intravenous injection. Amsler and Huber (1946) examined the anterior chamber at frequent intervals through the corneal microscope after the intravenous injection of fluorescein, the focal beam from the slit lamp directed into the eye being reduced in intensity by means of a resistance in the lamp circuit until the fluorescence of the aqueous was just imperceptible. This is regarded as a measure of the concentration of fluorescein in the aqueous at that particular time, the degree of fluorescence of a solution within the concentrations studied, being taken as inversely proportional to the quantity of light required to make it just perceptible, although the relationship is not linear. Goldmann (1949), on the other hand, assessed the concentration of fluorescein in the aqueous at frequent intervals by adjusting a standard fluorescent beam so that it matched the fluorescence of the aqueous observed simultaneously through a corneal microscope.

The instrument described above has been designed to record objectively the relative changes in concentration of a fluorescent substance in the anterior chamber of the eye, and is of value not only in detecting gross differences in the permeability of the blood-aqueous barrier clinically, but also in studying the wider problems of the flow of aqueous humour experimentally; it is of particular value in animal studies when high concentrations of the dye may be used. The interpretation of the results is based on the observation that the intensity of fluorescence, and in consequence the output

*The state of dynamic equilibrium differs from that of chemical equilibrium in that a balance is reached between a
substance on either side of a living membrane without necessarily inducing the same concentration of that substance substance on either 
of the photo-electric multiplier tube, is directly proportional to the concentration of fluorescein. This relationship was determined by measuring with the fluorophotometer the fluorescence of various solutions containing a known concentration of fluorescein. These solutions, placed in a squareshaped cell of fused silica glass were illuminated by the focal light from the slit lamp, and observed with the fluorophotometer through one of the cell faces lying parallel with the light beam. It will be noted that over a wide range of concentration of fluorescein the relationship

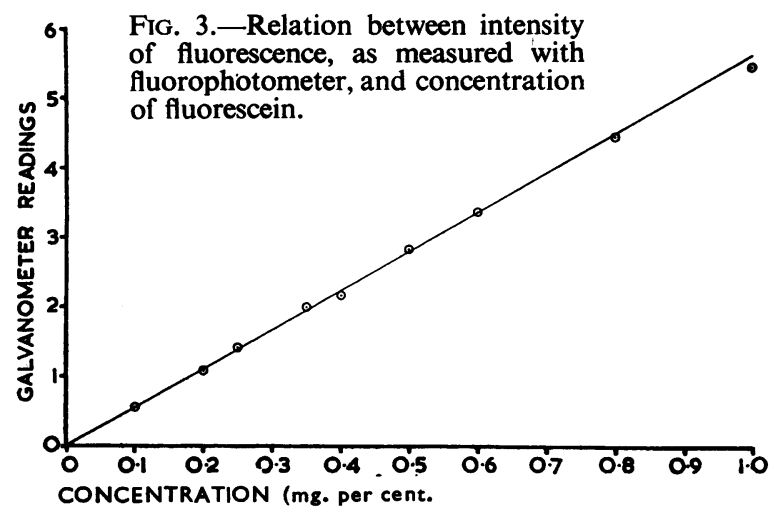
is strictly linear (Fig. 3).

An attempt has been made with this apparatus to overcome certain of the difficulties encountered in an approach to this problem. First, by limiting the effective field of view to a constant area of the anterior chamber throughout the period of the experiment, any inconsistencies due to an uneven concentration of the dye in the aqueous are largely eliminated. Secondly, by being able to record the concentration of the fluorescein within the aqueous at frequent intervals without disturbing the integrity of the blood-aqueous barrier, the changes in the concentration of the dye are obtained over the complete time-course. These results may then be correlated with the timecourse of the dye in the plasma, and from this the constants of penetration and exit, and the rate of flow of aqueous humour may be calculated.

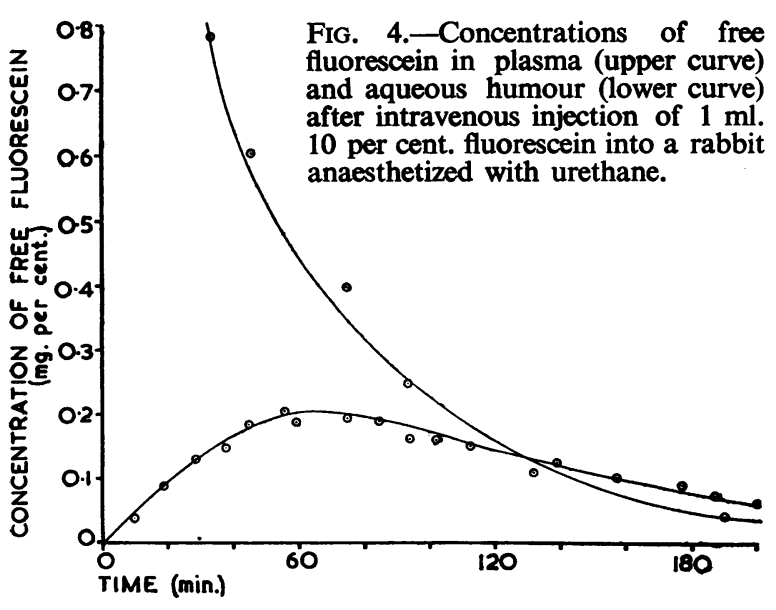

There are, however, certain disadvantages in this approach. First, there are not many compounds which are suitable for such a study, fluorescein and eosein being two of the few dyes which may be used in appropriate concentrations. Secondly, since a high proportion of these dyes become bound to the plasma proteins, to which the normal bloodaqueous barrier is almost 
impermeable, only that fraction of the dye which is not bound or adsorbed in this way diffuses into the aqueous humour. Furthermore, it is possible that some of the free dye in the aqueous may also be lost by becoming bound to the proteins of the tissues lining the anterior chamber.

An example of the type of results which may be obtained in the rabbit after intravenous injection of $1 \mathrm{ml}$. of a 10 per cent. solution of fluorescein is shown in Fig. 4. The rabbit is maintained in position throughout the period of the experiment in a specially designed cradle attached to the table of the slit lamp (see Fig. 2). Samples of blood are taken at periodic intervals, and analysed for total and freely diffusible fluorescein. The degree of fluorescence in the aqueous humour is measured by the fluorophotometer. At the end of the experiment the aqueous is removed and analysed for fluorescein, and by correlating this analysis with the final fluorophotometric reading it is possible to translate the other readings into absolute values.

\section{Summary}

(1) A description is given of a simple apparatus which fits on to a conventional Haag-Streit slit lamp and enables objective measurements to be made of the degree of fluorescence in the anterior chamber of the living eye.

(2) There is a linear relationship between the concentration of fluorescein in a solution and the degree of fluorescence of the solution as measured by the fluorophotometer over a wide range of concentrations of fluorescein.

(3) The application of this technique to dynamic studies of the formation and outflow of the aqueous humour in animals and man is considered.

We should like to thank Sir Stewart Duke-Elder for his help and interest during the course of this work, and the Medical Research Council for defraying the cost of the research. We are also indebted to Messrs. N. Roberts and A. Church for the assembly of the fluorophotometer.

\section{REFERENCES}

AMSLeR, M., and Huber, A. (1946). Ophthalmologica (Basel), 111, 155.

BÁrÁNY, E., and KINSEY, V. E. (1949). Amer. J. Ophthal., 32, June (pt. 2), 177.

EHRLICH, P. (1882). Dtsch. med. Wschr., 8, 54.

GoldMANN, H. (1949). Ophthalmologica (Basel), 117, 240.

MAURICE, D. M. (1951). J. Physiol. (Lond.), 112, 367.

PaLM, E. (1948). Acta ophthal. (Kbh.), Suppl. 32. 